\title{
Combined effects of double nonlocal terms in the nonlinear eigenvalue problems
}

\author{
Shuai $\mathrm{Yao}^{1}$ and Haibo Chen ${ }^{2}$ \\ ${ }^{1}$ Central South University School of Mathematics and Statistics \\ ${ }^{2}$ Department of Mathematics, Central South University
}

February 22, 2022

\begin{abstract}
In this paper, we study the following eigenvalue problem for Kirchhoff type equation with Hartree nonlinearity: \begin } \{ \text { equation\} }

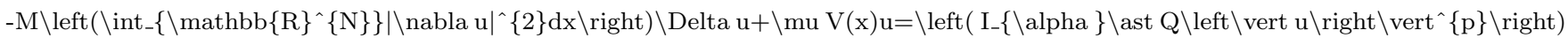
$\mathrm{Q} \backslash$ vert $\mathrm{u} \backslash$ vert ${ }^{\wedge}\{\mathrm{p}-2\} \mathrm{u}+\backslash$ lambda $\mathrm{f}(\mathrm{x}) \mathrm{u} \backslash$ quad $\backslash \operatorname{text}\{\operatorname{in}\} \backslash$ quad $\backslash \operatorname{mathbb}\{\mathrm{R}\}^{\wedge}\{\mathrm{N}\}, \backslash$ end $\{$ equation $\}$ where $\$ \mathrm{~N} \backslash$ geq $3, \mathrm{a}, \backslash \mathrm{mu}>00 \$$ parameters, $\$ \mathrm{M}(\mathrm{t})=\mathrm{at}+1 \$, \$ \mathrm{~V} \backslash$ in $\mathrm{C}\left(\backslash \operatorname{mathbb}\{\mathrm{R}\}^{\wedge}\{\mathrm{N}\}, \backslash\right.$ mathbb $\left.\{\mathrm{R}\} \%^{\wedge}\{+\}\right) \$, \$ \mathrm{I}_{-}\{\backslash$ alpha $\} \$$ is the Riesz potential, $\$ \mathrm{Q}(\mathrm{x}) \backslash$ in $\mathrm{L}^{\wedge}\{\backslash \operatorname{infty}\}\left(\backslash \operatorname{mathbb}\{\mathrm{R}\}^{\wedge}\{\mathrm{N}\}\right) \$$ with changes sign in $\$ \backslash$ overline $\{\backslash$ Omega $\}:=\backslash \operatorname{left} \backslash\{\mathrm{V}(\mathrm{x})=0 \backslash$ right $\backslash\} \$$, and $\$ 0$
\end{abstract}

\section{Hosted file}

YC-MMA-submission.pdf available at https://authorea.com/users/461817/articles/557423combined-effects-of-double-nonlocal-terms-in-the-nonlinear-eigenvalue-problems 